\title{
REFRACTORY ACPA-POSITIVE JUVENILE IDIOPATHIC ARTHRITIS: SUCCESSFUL TREATMENT WITH RITUXIMABE
}

\begin{abstract}
Júlia Boechat Farani ${ }^{1, \star}$, Erika Biegelmeyer ${ }^{1}$, Evelise Mileski do Amaral Berlet ${ }^{1}$, Marcel Mathias Villaça ${ }^{1}$, Marília Voges de Souza ${ }^{1}$, Mauro Waldemar Keiserman ${ }^{1}$, Maria Mercedes Caracciolo Picarelli ${ }^{1}$
\end{abstract}

1.Pontifícia Universidade Católica do Rio Grande do Sul, Porto Alegre (RS), Brazil.

*Corresponding author: juliaboechat@globo.com

\section{BACKGROUND}

Juvenile idiopathic arthritis (JIA) might lead to continuous inflammatory activity and severe disabilities in adult life. Anticitrullinated protein antibody (ACPA) positivity may correlate with poor outcomes in adulthood. B-cell depletion with rituximab is an alternative treatment for refractory JIA.

\section{CASE REPORT}

An 11-year-old white male patient presented to our clinic with a previous diagnosis of JIA. Symptoms had begun at the age of 7 years, presenting with oligoarthritis of the right knee and left wrist and progressing to symmetric polyarthritis of knees, wrists and ankles over four years. There was passive tobacco smoke exposure in infancy, allergic rhinitis and recurrent upper respiratory tract infections from 2 to 5 years old. He also had chronic diarrhea and intermittent abdominal pain since the age of 6 years, when he was diagnosed with coeliac disease, which improved with a gluten-free diet. There was no history of fever, cutaneous rash, axial symptoms, uveitis, enthesitis, dactylitis or psoriasis. Familial history was also negative for autoimmune diseases. At presentation, there was an erythrocyte sedimentation rate (ESR) of $120 \mathrm{~mm} / \mathrm{h}$ and a C-reactive protein (CRP) of $48 \mathrm{mg} / \mathrm{dL}$. Rheumatoid factor, antinuclear antibodies and viral serologies were repeatedly negative. Right-knee ultrasound and magnetic resonance confirmed chronic synovitis and joint effusion. He had been treated with methotrexate and leflunomide with a poor response, as well as anti-TNF therapy with adalimumab, with secondary failure over 6 months. The diagnosis was revisited. Arthrocentesis of the right knee revealed inflammatory arthritis with negative cultures. Arthroscopy with synovial biopsy of the right knee revealed chronic edematous synovitis with fibrosis, without granuloma or chronic infections. Finally, ACPA was positive in high titers of 101 (reference value $<4$ ). Treatment was changed, but the disease was also refractory to TNF inhibitor etanercept (secondary failure over 6 months), anti-IL-6-receptor therapy with tocilizumab (primary failure) and selective T-cell co-stimulation with abatacept (secondary failure over 9 months). There was relapsing arthritis of hands and wrists, Cushing's syndrome, and obesity due to chronic corticoid therapy. Finally, B-cell depletion therapy with rituximab was initiated, $1 \mathrm{~g}$ on day 1 and $1 \mathrm{~g}$ on day 15 , which was well tolerated and led to a complete articular response and gradual glucocorticoid-dose tapering over 3 months.

\section{CONCLUSION}

This is a challenging case of extended oligoarticular JIA. ACPA positivity, tobacco smoke exposure in infancy and dysbiosis due to coeliac disease might have contributed to its refractoriness. Rituximab was an interesting treatment option.

\section{KEYWORDS}

Juvenile idiopathic arthritis, Refractoriness, Rituximab. 